\title{
A virus-induced molecular mimicry model of multiple sclerosis
}

\author{
Julie K. Olson, ${ }^{1}$ J. Ludovic Croxford, ${ }^{1}$ Miriam. A. Calenoff, ${ }^{2}$ Mauro C. Dal Canto, ${ }^{2}$ \\ and Stephen D. Miller ${ }^{1}$
}

${ }^{1}$ Department of Microbiology-Immunology and the Interdepartmental Immunobiology Center, and

${ }^{2}$ Department of Pathology, Northwestern University Medical School, Chicago, Illinois, USA

Address correspondence to: Stephen D. Miller, Department of Microbiology-Immunology,

Northwestern University Medical School, 303 East Chicago Avenue, Chicago, Illinois 60611, USA.

Phone: (312) 503-7674; Fax: (312) 503-1154; E-mail: s-d-miller@northwestern.edu.

Julie K. Olson and J. Ludovic Croxford both contributed significantly to this work.

Received for publication April 13, 2001, and accepted in revised form June 4, 2001.

\begin{abstract}
Molecular mimicry is the process by which virus infection activates $\mathrm{T}$ cells that are cross-reactive with self antigens. Infection of $\mathrm{SJL} / \mathrm{J}$ mice with the neurotropic picornavirus Theiler's murine encephalomyelitis virus (TMEV) leads to a progressive $\mathrm{CD}^{+}{ }^{+} \mathrm{T}$ cell-mediated demyelinating disease similar to multiple sclerosis. To study the potential of virus-induced molecular mimicry to initiate autoimmune demyelination, a nonpathogenic TMEV variant was engineered to encode a 30-mer peptide encompassing the immunodominant encephalitogenic myelin proteolipid protein (PLP139-151) epitope. Infection with the PLP139-151-encoding TMEV led within 10-14 days to a rapid-onset paralytic demyelinating disease characterized by PLP139-151-specific CD4 ${ }^{+}$Th1 responses; insertion of a nonself ovalbumin sequence led to restoration of the normal late-onset disease. Early-onset disease was also observed in mice infected with a TMEV encoding PLP139-151 with an amino acid substitution at the secondary $\mathrm{T}$ cell receptor (TCR) contact residue (H147A), but not in mice infected with TMEV encoding a PLP139-151 substitution at the primary TCR contact (W144A). Most significantly, mice infected with TMEV encoding a Haemophilus influenzae mimic peptide, sharing only 6 of 13 amino acids with PLP139-151, displayed rapid-onset disease and developed cross-reactive PLP139-151-specific CD4 ${ }^{+}$Th1 responses. To our knowledge, this is the first study showing that a naturally infectious virus encoding a myelin epitope mimic can directly initiate organ-specific $T$ cell-mediated autoimmunity.
\end{abstract}

J. Clin. Invest. 108:311-318 (2001). DOI:10.1172/JCI200113032.

\section{Introduction}

Multiple sclerosis (MS) is an autoimmune demyelinating disease characterized by myelin epitope-specific $\mathrm{CD}^{+} \mathrm{T}$ cells infiltrating the central nervous system (CNS) $(1,2)$. The mechanism(s) underlying the initiation and progression of MS is not well understood; however, epidemiologic studies have provided strong suggestive evidence for a role for virus infection(s) in its development and/or exacerbation (3). Postulated mechanisms for infection-induced autoimmunity include: activation of autoreactive $\mathrm{T}$ cells secondary to an encounter with a pathogen by epitopes shared or cross-reactive with self antigens, i.e., molecular mimicry $(4,5)$; de novo activation of autoreactive $\mathrm{T}$ cells by sequestered antigens released secondary to tissue destruction mediated by virus-specific $\mathrm{T}$ cells, i.e., epitope spreading (6-8); and stimulation of autoreactive $T$ cells bearing particular $V \beta$ receptors by virus-encoded superantigens (9).

Molecular mimicry remains the major postulated mechanism by which infections may trigger autoimmune tissue damage $(4,10)$. Evidence for molecular mimicry stems mainly from experiments showing that mice expressing virus proteins as transgenes develop autoimmune disease after virus infection $(11,12)$ and from reports showing the considerable degeneracy of the $T$ cell recognition wherein viral peptides sharing limited sequence homology with self peptides can stimulate autoreactive $\mathrm{T}$ cells in vitro $(10,13,14)$. More recently, $\mathrm{T}$ cell clones have been used to identify mimic epitopes in Borrelia burgdorferi, which may be potentially involved in the pathogenesis of Lyme arthritis and neuroborreliosis $(15,16)$, and immunization of mice with a Chlamydia pneumoniae peptide was shown to induce heart disease via antigenic mimicry of a heart-muscle protein (17). However, more direct evidence stems from a recent report demonstrating that infection of mice with herpes simplex virus type I leads to the development of herpes stromal keratitis, an autoimmune eye disease, in which $\mathrm{CD}^{+} \mathrm{T}$ cells reactive to a viral coat protein cross-react with an unidentified corneal antigen (18).

Theiler's murine encephalomyelitis virus (TMEV) is an endogenous mouse pathogen that normally establishes a lifelong persistent CNS infection and leads to the development of a progressive $\mathrm{CD} 4^{+} \mathrm{T}$ cell-mediated demyelinating disease that shares pathologic and immunologic similarities with human MS $(19,20)$. Clinical disease in SJL mice infected with wild-type TMEV-induced demyelinating disease normally devel- 
ops 30-35 days postinfection (PI) and is initiated by virus-specific Th1 cells targeting CNS-persistent virus, leading to bystander myelin damage (21). Initial myelin destruction eventually results (50-60 days PI) in the induction of autoreactive $\mathrm{T}$ cells (via epitope spreading) specific for proteolipid protein epitope PLP139-151 (22).

In the current study, we used a unique approach to test the efficacy of molecular mimicry in initiating CNS autoimmunity through viral infection by inserting native or mimic sequences of PLP139-151 into a nonpathogenic TMEV variant and testing for induction of CNS disease. The results clearly show that PLP139151-specific $\mathrm{CD}^{+} \mathrm{T}$ cell responses and autoimmune demyelination are initiated by infection of SJL mice with TMEV encoding native PLP139-151, a PLP139-151 altered peptide ligand, or a PLP139-151 sequence mimic encoded in the protease IV protein of Haemophilus influenzae in the course of a natural virus infection.

\section{Methods}

Construction of mimic BeAn viruses. The cDNA encoding the BeAn strain of TMEV was modified to contain a ClaI restriction site at bp 1137, resulting in the deletion of 23 amino acids from the virus leader (designated $\Delta \mathrm{ClaI}-$ BeAn). PCR methods were used to insert ClaI sites into PLP DNA at both ends of a 30-amino acid piece, PLP130-159, which encompassed the encephalitogenic PLP139-151 epitope (Figure 1a). This piece was then inserted into the ClaI site in the $\triangle$ ClaI-BeAn virus cDNA. Mimic sequences of PLP139-151 were constructed by PCR mutagenesis of the PLP DNA prior to insertion into the $\Delta$ ClaI-BeAn viral genome to introduce amino acid substitutions individually at position $144(\mathrm{~W} \rightarrow \mathrm{A})$ or position $147(\mathrm{H} \rightarrow \mathrm{A})$, or collectively at positions 139 $(\mathrm{H} \rightarrow \mathrm{E}), 140(\mathrm{C} \rightarrow \mathrm{Q}), 142(\mathrm{G} \rightarrow \mathrm{V}), 147(\mathrm{H} \rightarrow \mathrm{L}), 149$ $(\mathrm{D} \rightarrow \mathrm{A}), 150(\mathrm{~K} \rightarrow \mathrm{P})$, and $151(\mathrm{~F} \rightarrow \mathrm{I})$ corresponding to a mimic naturally expressed by $H$. influenzae (HI) protease. To produce a non-self epitope-containing virus, ovalbumin peptide OVA317-346, encompassing the OVA323339 epitope, was inserted into $\triangle$ ClaI-BeAn. Viral RNA was produced from the cDNA for each recombinant using a T7 promoter transcription kit (Roche Molecular Biochemicals, Indianapolis, Indiana, USA) and then transfected using Lipofectin (Life Technologies Inc., Rockville, Maryland, USA) into BHK-21 cells to produce infectious virus. BHK-21 cells were repeatedly infected with virus in serum-free DMEM medium (Sigma Chemical Co., St. Louis, Missouri, USA) until a viral titer of 1 $\times 10^{9} \mathrm{PFU} / \mathrm{ml}$ was obtained for each viral construct.

Infection of mice. Five- to 6-week old female SJL mice were purchased from Harlan Laboratories (Bethesda, Maryland, USA) and housed in the Northwestern University animal facility. Mice were infected by intracerebral injection with $3 \times 10^{7}$ PFU of virus: PLP139-BeAn, H147A PLP139-BeAn, W144A PLP139-BeAn, $\Delta$ ClaI-BeAn, OVA323-BeAn, HI-BeAn, or BeAn strain 8386 (wild-type). Mice were scored at daily intervals on a clinical scale of $0-5$ : 0 , mice show no signs of disease; 1 , mice show mild gait abnormalities; 2 , mice show more severe gait abnor- malities; 3 , mice have paralysis in one limb; 4 , mice have more than one paralyzed limb; 5 , mice are moribund.

Peptides. PLP139-151(HSLGKWLGHPDKF), PLP56-70 (DYEYLINVIHAFQYV), PLP178-191(NTWTTCQSIAFPSK), VP2 70-86 (WTTSQEAFSHIRIPLPH), and HI574586 (EQLVKWLGLPAPI) were purchased from Peptides International (Louisville, Kentucky, USA). The amino acid composition was verified by mass spectrometry, and purity was assessed by HPLC.

Delayed-type hypersensitivity response. Delayed-type hypersensitivity (DTH) assays were performed by injecting mice subcutaneously with $5 \mu \mathrm{g}$ of the challenge peptide, PLP139-151, or VP2 70-86, into the ears. The ear thickness was determined before injection using a Mitutoyo model 7326 engineer's micrometer (Schlesinger's Tools, Brooklyn, New York, USA). After 24 hours, the ears were remeasured and differences in ear swelling over prechallenge thickness were expressed in units of $10^{-4}$ inches \pm SEM.

$T$ cell proliferation and cytokine assays. Spleens were removed from infected mice at various times following infection. Splenocytes were cultured at $1 \times 10^{6}$ cells per well (in 96-well microtiter plates) in HL-1 medium (BioWhittaker Inc., Walkersville, Maryland, USA) supplemented with $2 \mathrm{mM}$ L-glutamine, $100 \mathrm{U} / \mathrm{ml}$ penicillin, $100 \mu \mathrm{g} / \mathrm{ml}$ streptomycin. Peptides were added to the wells at increasing concentrations from 10 to $100 \mu \mathrm{M}$. Plates were incubated at $37^{\circ} \mathrm{C}$ for 72 hours and then pulsed with $1 \mu \mathrm{Ci}^{3}[\mathrm{H}]-\mathrm{TdR}$ for 24 hours before harvesting and counting. Proliferation was determined with triplicate wells for each peptide concentration and then expressed as $\mathrm{cPm} \pm \mathrm{SEM}$ and as a stimulation index in comparison with PBS-containing wells. For cytokine analysis, a duplicate set of proliferation wells was used to collect supernatants at 48 and 72 hours. Supernatants were analyzed for IL-2, IFN- $\gamma$, TNF- $\alpha$, or IL- 4 secretion by ELISA assays (Pierce Endogen, Rockford, Illinois, USA).

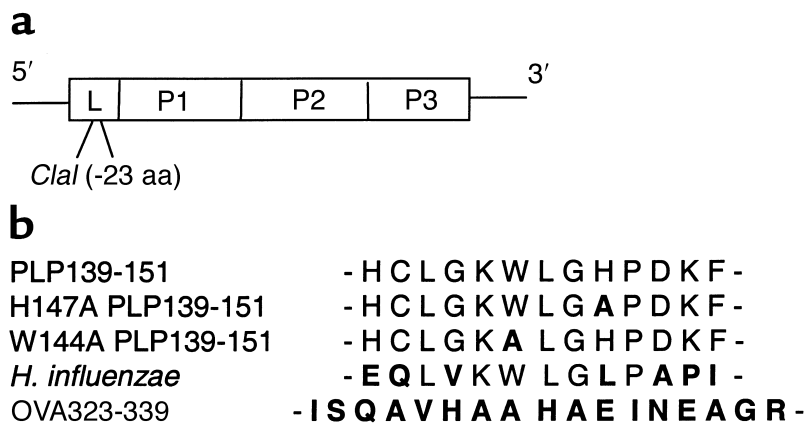

\section{Figure 1}

Construction of TMEV containing sequences for PLP139-151 and molecular mimics of PLP139-151. (a) cDNA from TMEV strain BeAn 8386 had a Clal restriction site inserted into the leader sequence resulting in a 23-amino acid deletion. (b) Thirty-amino acid sequences $(24,27)$ encompassing the native immunodominant myelin epitope PLP139-151; H147A- and W144A-substituted PLP139-151 sequences; a PLP139-151 mimic epitope from $H$. influenzae; or OVA323-339 control peptide were inserted into the Clal restriction site in the viral cDNA. Viral RNA was synthesized and infectious virus was produced in BHK-21 cells. 


\section{Results}

Mice infected with PLP139-TMEV develop early-onset inflammatory demyelinating disease. A 30-amino acid sequence (PLP130-159) encompassing the native encephalitogenic PLP139-151 peptide was inserted into the leader portion of a cDNA construct of a nonpersisting, mutant BeAn strain of TMEV that had a ClaI restriction site added and 23 amino acids deleted ( $\triangle$ ClaI-BeAn) (Figure 1a). In addition, recombinant PLP139-151 epitope mimic viruses were constructed (Figure $1 \mathrm{~b}$ ) that had amino acid substitutions at either the primary (W144A) or secondary (H147A) T cell receptor (TCR) recognition sites $(23,24)$. As expected, SJL mice infected intracerebrally with the wild-type BeAn strain of TMEV (WT BeAn) developed clinical signs of demyelinating disease around day 30-35 PI and exhibited a chronic-progressive demyelinating disease (Figure 2, a and b). Clinical signs begin with an abnormal gait and worsen to paralysis and often death. Mice infected with the $\Delta \mathrm{ClaI}-\mathrm{BeAn}$ virus, the nonpersisting parental construct for the recombinant viruses, did not develop any signs of autoimmune demyelinating disease. Interestingly, insertion of a 30-mer encompassing a non-self OVA323-339 epitope led to clinical disease, albeit with a delayed onset (approximately 50 days PI) compared with the wild-type BeAn TMEV strain, indicating that reintroduction of a nonspecific 30-mer into the leader of the $\Delta$ ClaI-BeAn parental strain restores the ability of the virus to persist in vivo. Most significantly, mice infected with PLP139-BeAn virus developed severe clinical signs at very early times, i.e. 7-10 days PI. A similar early-onset disease course was observed in mice infected with the H147A PLP139-BeAn mimic virus. In contrast, mice infected with the W144A PLP139-BeAn, containing an amino acid substitution at the primary TCR contact residue, exhibited a delayed disease course similar to that of the virus containing the non-self
OVA323-339 epitope. Virus load in the brains and spinal cords was similar up to 28 days after infection with any of these strains (data not shown), demonstrating that the recombinant viruses were able to persist in the CNS at wild-type levels during the early stages of infection. Spinal cords from mice sacrificed at 14 days PI with PLP139-BeAn virus had areas of infiltrating immune cells and signs of demyelination (Figure 2d), while mice infected with OVA323-BeAn virus had no signs of infiltration or demyelination (Figure 2c). Histology in the spinal cords of mice infected with H147A PLP139-BeAn was similar to that observed in PLP139-BeAn infected mice, containing infiltrating cells and displaying moderate demyelination, while mice infected with W144A PLP139-BeAn virus did not show histologic signs of disease at 14 days PI (data not shown). Thus, mice infected with viruses containing either the native encephalitogenic PLP139-151 epitope or the H147A altered peptide ligand (APL) developed early-onset demyelinating disease, while the W144A PLP139-BeAn virus APL was unable to initiate the early-onset demyelination.

Mice developing demyelinating disease display PLP139151-specific $\mathrm{CD}^{+} \mathrm{T}$ cell responses. The infected mice were next analyzed to determine the temporal progression of antigen-specific $\mathrm{T}$ cell responses generated during the infection. Mice infected with the wild-type strain of TMEV developed initial CD $4^{+}$DTH and T cell proliferative response to the immunodominant virus epitope, VP2 70-86, within 14 days after infection (Figure 3, a and b), which persisted through day 28 (Figure $3 \mathrm{c}$ ) and day 90 (Figure 3d). As previously reported $(22,25)$, myelinspecific $\mathrm{CD} 4^{+} \mathrm{T}$ cell responses in mice infected with wildtype TMEV are first observed to the immunodominant PLP139-151 epitope beginning around 50 days PI and eventually spread to other myelin epitopes, including PLP56-70 and PLP178-191, by 120-150 days PI. This is

\footnotetext{
Figure 2

SJL mice infected with TMEV containing native PLP139151 sequence or sequence mimics develop early demyelinating disease. Separate groups of SJL mice $(n=10)$ were injected intracerebrally with $3 \times 10^{7} \mathrm{PFU}$ of the indicated recombinant viruses as well as wild-type virus (WT BeAn) or the parental construct $(\Delta \mathrm{Clal}-\mathrm{BeAn})$. The mean clinical score (a) based on a grading scale of $0-5$ and the percentage of mice showing clinical signs of demyelination (b) were determined at varying times after infection. Results are representative of at least three separate experiments. At 14 days $\mathrm{PI}$, mice infected with OVA323-BeAn (c) or PLP139-BeAn (d) were anesthetized and sacrificed by total body perfusion through the left ventricle using chilled $3 \%$ glutaraldehyde in PBS, $\mathrm{pH}$ 7.3. Spinal cords were cut into $1-\mu \mathrm{m}$-thick segments and post-fixed in $\mathrm{OsO}_{4}$, dehydrated, and embedded in Epon. Toluidine blue-stained sections from ten segments per mouse were read and scored blind.
}

a

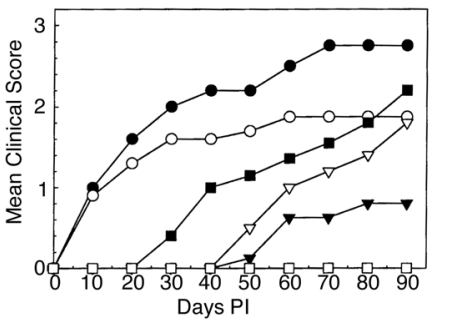

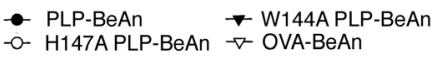

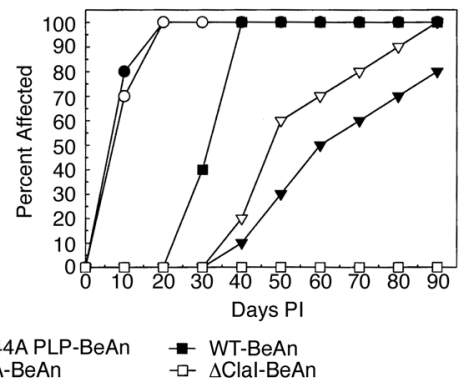

C

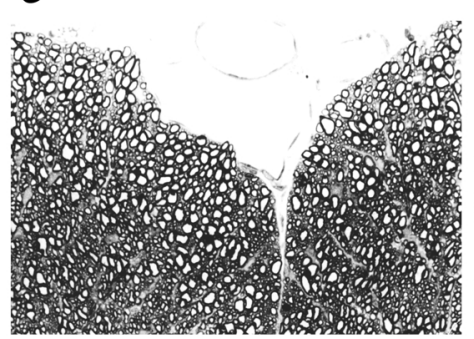

d

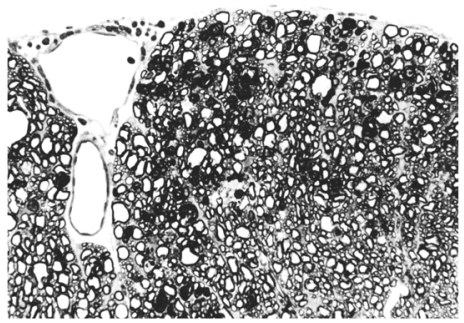



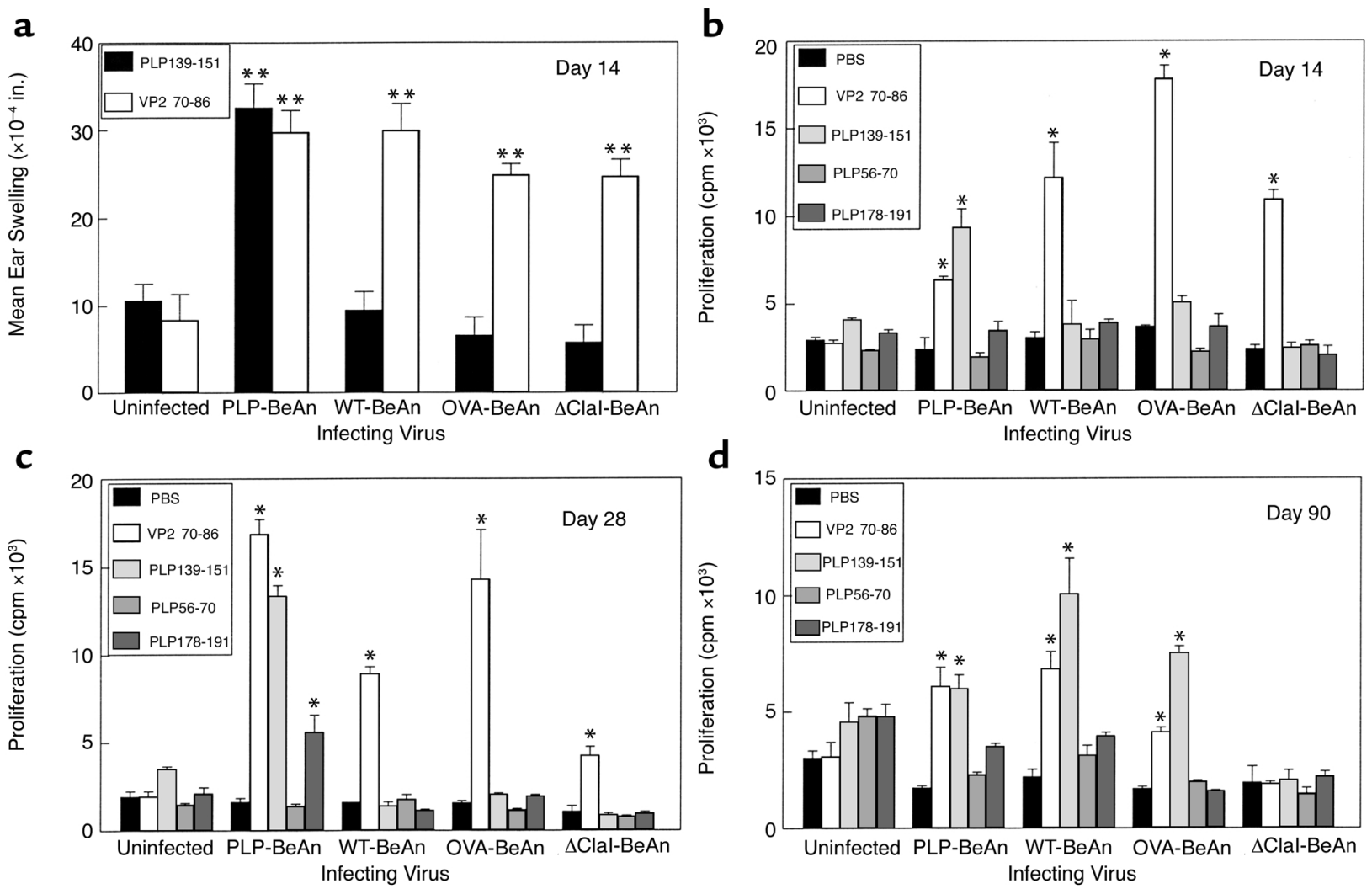

Figure 3

SJL mice infected with TMEV containing the native PLP139-151 sequence develop early myelin-specific CD4 ${ }^{+} T$ cell responses. SJL mice were infected with PLP139-BeAn, wild-type BeAn, OVA323-BeAn, or $\Delta$ Clal-BeAn. (a) At 14 days PI, the mice were ear-challenged with $5 \mu \mathrm{g}$ PLP139151 or TMEV VP2 70-86 and in vivo DTH responses were determined 24 hours after challenge. ** DTH responses significantly higher than those in mock infected controls; $P \leq 0.01$. Splenic CD4 ${ }^{+} T$ cell proliferative responses from $2-3$ mice per group were determined at 14 days (b), 28 days (c), and 90 days PI (d) to the immunodominant TMEV epitope, VP2 70-86, and to a panel of myelin epitopes - PLP139-151, PLP56-70, and PLP178-191. T cell proliferation was determined at 96 hours and results expressed as mean cpm \pm SEM of triplicate cultures

*Stimulation index $(\mathrm{cpm}+$ antigen $) /(\mathrm{cpm}-$ antigen $) \geq 3$. Results are representative of three separate experiments.

confirmed by the presence of PLP139-151-specific splenic $\mathrm{T}$ cell proliferative responses at 90 days PI (Figure 3d), but not at day 14 (Figure 3b) or 28 (Figure 3c), in these mice. In contrast, mice infected with the PLP139-BeAn virus developed early $\mathrm{CD}^{+} \mathrm{T}$ cell responses to both VP2 70-86 and the virus-encoded PLP139-151 epitope by 14 days PI (Figure 3, a and b), which each persisted through day 90 (Figure $3, \mathrm{c}$ and $\mathrm{d}$ ). This indicates that the PLP139-151 peptide could be processed from the 30-mer insertion within the virus leader polyprotein and that the early-onset demyelinating disease is temporally related to the autoimmune response. Interestingly, mice infected with $\Delta$ ClaI-BeAn virus had only virus-specific $\mathrm{CD}^{+} \mathrm{T}$ cell responses early after infection (Figure 3 , $a$ and $b$ ) and these responses waned by 90 days PI (Figure 3d), indicative of the failure of this variant to persist in the CNS (data not shown). Infection with OVA323-BeAn expressing the non-self antigen resulted in a $\mathrm{CD}^{+} \mathrm{T}$ cell response pattern similar to that observed in wild-type virus infection, where PLP139-151 responses were observed only at day $90 \mathrm{PI}$.

$\mathrm{CD}^{+} \mathrm{T}$ cell responses in mice infected with the H147A and W144A viruses differed significantly.
Although infection with either virus induced early and sustained responses to VP2 70-86, only mice infected with the H147A PLP139-BeAn virus, which developed early-onset demyelination (Figure 2, a and b), developed $\mathrm{CD} 4^{+} \mathrm{T}$ cell responses cross-reactive with PLP139151 at early times following infection (Figure 4, a and b). Interestingly, PLP178-191-specific T cell responses were observed at 90 days PI in mice infected with either PLP139-BeAn or the H147A APL virus (Figure $3 \mathrm{~d}$ and Figure $4 \mathrm{~d}$ ). Therefore, infection with TMEV expressing a self myelin peptide with an amino acid substitution at the secondary, but not the primary, TCR contact residue can initiate a $\mathrm{CD} 4^{+} \mathrm{T}$ cell response cross-reactive with the native PLP139-151 epitope. In addition, as PLP178-191-specific responses were observed at 28 and 90 days PI, it appears that sustained tissue damage initiated by virus-induced molecular mimicry can subsequently lead to epitope spreading to additional PLP epitopes following the release of endogenous epitopes. Infection with TMEV encoding the H147A APL induces PLP139-151-specific CD4+ Th1 responses. Autoimmune demyelination is associated with CNS infiltration of autoreactive proinflammatory $\mathrm{CD} 4^{+} \mathrm{Th} 1$ cells (26). 
We thus analyzed the cytokine profile of the antigenspecific $T$ cell responses at early ( 28 days) and late (90 days) time points in the infected mice. IL-2 secretion correlated with the virus- and myelin-specific $\mathrm{CD}^{+} \mathrm{T}$ cell proliferation profiles for the mice infected with the different virus constructs (Figure 5a). TMEV VP2 70-86-specific CD4 ${ }^{+} \mathrm{T}$ cells in all the infected mice secreted Th 1 (IFN- $\gamma$ and TNF- $\alpha$ ), but not Th2 (IL-4), cytokines at early and late times (Figure 5, b-d). PLP139-151-specific CD4+ $\mathrm{T}$ cells from mice infected with PLP139-BeAn and H147A PLP139-BeAn secreted IFN- $\gamma$ and TNF- $\alpha$ at 28 days PI, which was not seen in mice infected with W144A PLP139-BeAn or OVA323-BeAn viruses. However, by 90 days PI, PLP139-151-specific CD4 ${ }^{+} \mathrm{T}$ cells from all of the infected groups secreted IFN- $\gamma$ and TNF- $\alpha$. Thus, the PLP139-151-specific CD $4^{+} \mathrm{T}$ cells associated with the early-onset demyelinating disease in the mice infected with TMEV encoding either native PLP139-151 or the H147A APL were Th1 cells. IL-4 secretion was not demonstrated in any of the infected groups (Figure $5 \mathrm{~d}$ ).
Mice infected with TMEV expressing a bacterial mimic of PLP139-151 develop clinical disease associated with the crossreactive induction of $C D 4^{+} T h 1$ responses. A previous report by Carrizosa et al. (27) identified mimic peptides in mouse pathogens that cross-reacted with PLP139151-specific T cell clones derived from SJL mice. We inserted one of these mimic epitope sequences, $H$. influenzae protease IV 574-586 (HI574-586), which shares only 6 of 13 amino acids with the core PLP139-151 epitope (Figure 1b), into the BeAn viral genome. Significantly, mice infected with HI-BeAn developed early clinical disease following infection (Figure 6a). Clinical disease was somewhat less severe than in mice infected with PLP139BeAn, and disease onset was also slightly delayed. However, both PLP139-BeAn- and HI-BeAn-infected mice developed clinical signs at a significantly earlier time PI than did WT BeAn-infected mice. HI-BeAn disease was associated with the induction of cross-reactive PLP139151-specific $\mathrm{CD}^{+} \mathrm{T}$ cells as assessed by $\mathrm{T}$ cell proliferation assays (data not shown), and, significantly, $\mathrm{CD} 4^{+} \mathrm{T}$ cells at 21 days PI from both the PLP139-BeAn- and HIBeAn-infected mice secreted IFN- $\gamma$ at equivalent levels a

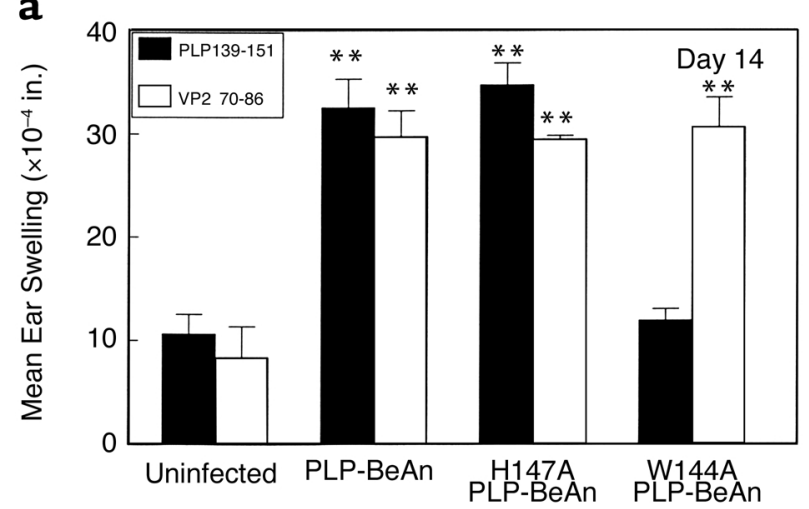

$\mathbf{C}$

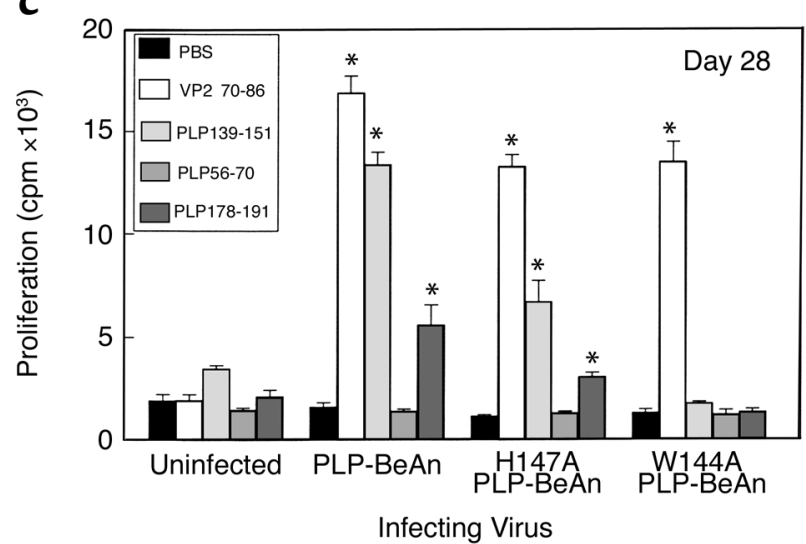

b

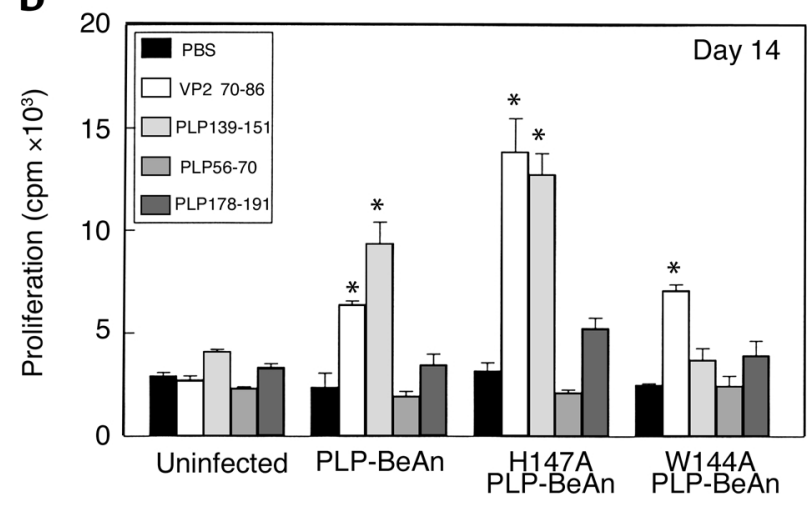

Infecting Virus

d

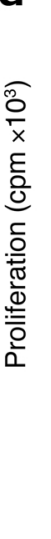

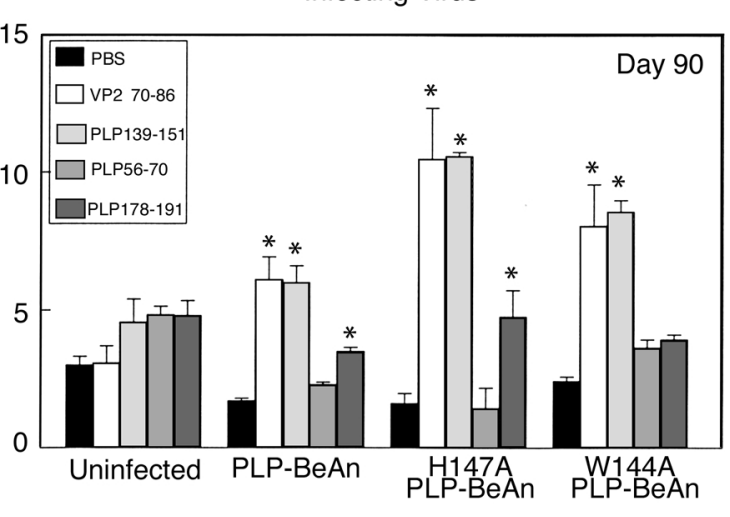

Infecting Virus

Figure 4

SJL mice infected with TMEV containing the H147A, but not the W144A, PLP139-151 APL develop early cross-reactive T cell responses to native PLP139-151. SJL mice were infected with PLP139-BeAn, H147A PLP139-BeAn, or W144A PLP139-BeAn. (a) At 14 days PI, the mice were earchallenged with $5 \mu \mathrm{g}$ PLP139-151 or VP2 70-86 and in vivo DTH responses were determined at 24 hours after challenge. ${ }^{*}$ DTH responses were significantly higher than those in mock-infected controls; $P \leq 0.01$. Splenic CD4+ $T$ cell proliferative responses from $2-3$ mice per group were determined at 14 days (b), 28 days (c), and 90 days PI (d) to the immunodominant TMEV epitope, VP2 70-86, and to a panel of self myelin epitopes - PLP139-151, PLP56-70, and PLP178-191. T cell proliferation was determined at 96 hours and results expressed as mean cpm \pm SEM of triplicate cultures. *Stimulation index $(\mathrm{cpm}+$ antigen $) /(\mathrm{cpm}-$ antigen $) \geq 3$. Results are representative of three separate experiments. 
a

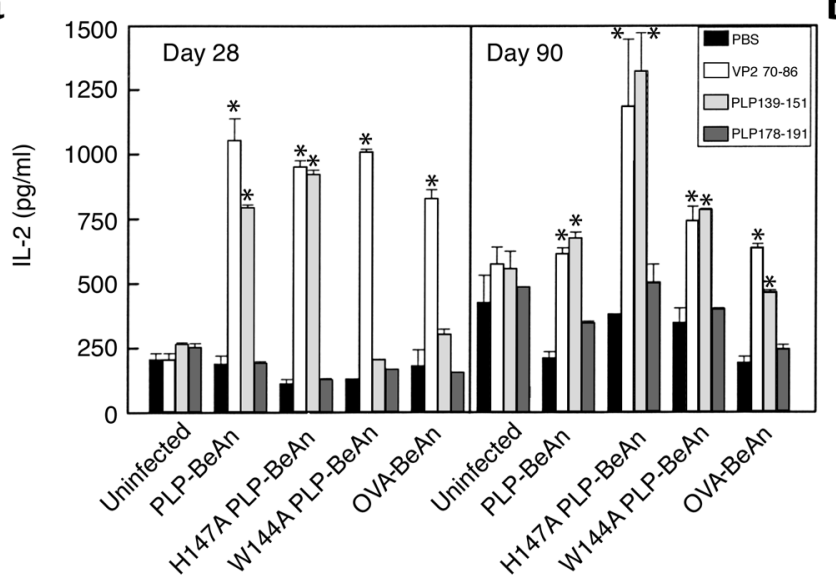

b

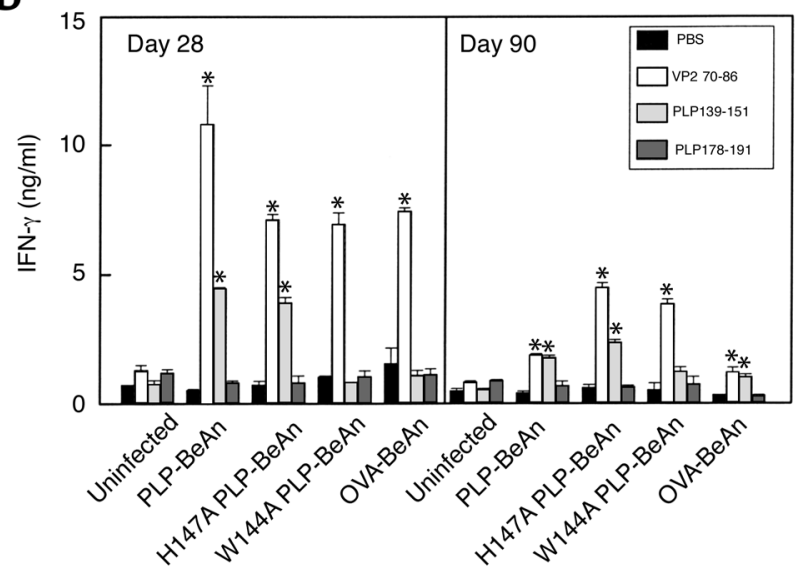

C

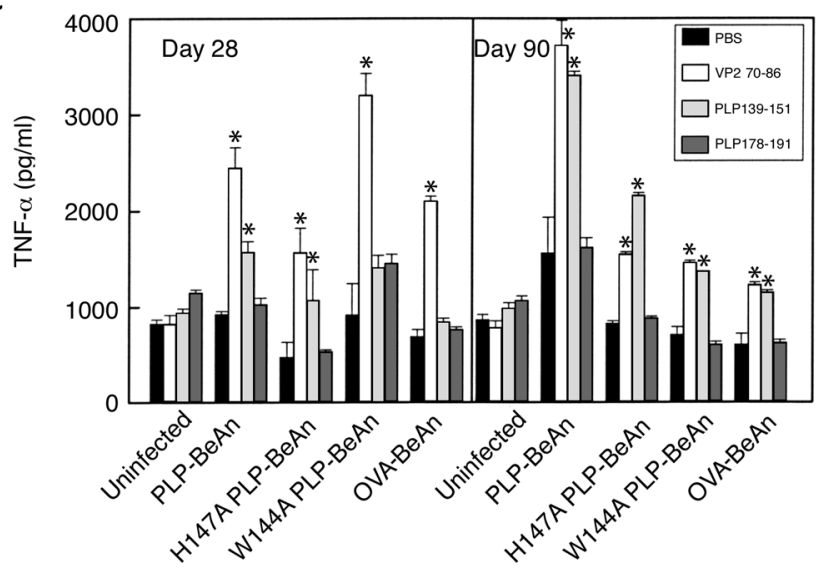

d

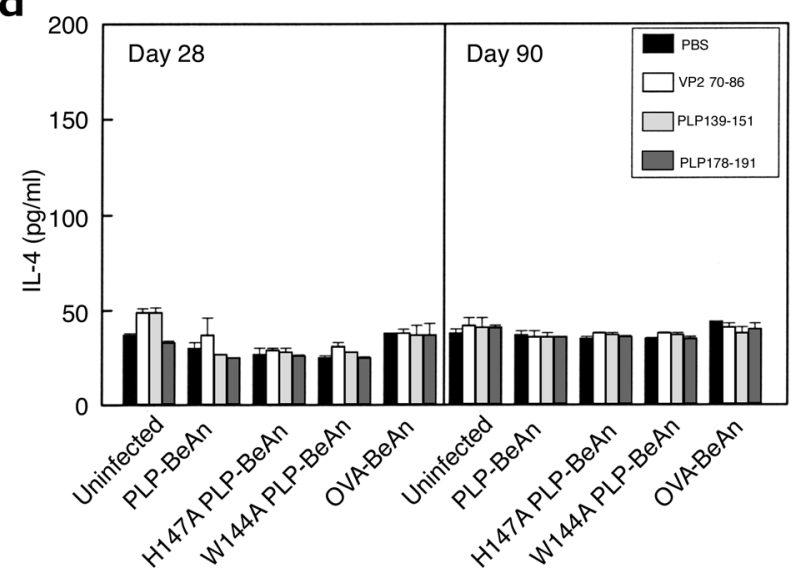

Figure 5

SJL mice infected with PLP139-BeAn and H147A PLP139-BeAn develop CD4+ Th1 responses to both virus and native PLP139-151 epitopes. Culture supernatants from $T$ cell proliferation assays shown in Figures 2 and 3 were collected at 48 hours and measured for cytokine secretion by ELISA: (a) IL-2, (b) IFN- $\gamma,(\mathbf{c})$ TNF- $\alpha$, and (d) IL-4. * Responses significantly above those of PBS-stimulated wells; $P \leq 0.01$.

upon challenge with the native PLP139-151 epitope (Figure 6b). Additionally, both PLP139-BeAn- and HIBeAn-infected mice secreted IFN- $\gamma$ when rechallenged with the immunodominant TMEV VP2 70-86 epitope.

\section{Discussion}

These studies clearly demonstrate that intracerebral infection with TMEV expressing a 30-mer encompassing the encephalitogenic self myelin PLP139-151 epitope can induce an early-onset, immune-mediated demyelinating disease in mice due to the rapid activation of PLP139151-specific $\mathrm{CD}^{+}$Th 1 cells. More significantly, a virus expressing an APL of this myelin epitope containing an amino acid substitution at the secondary TCR contact residue (H147A PLP139-BeAn) developed the early-onset demyelinating disease concomitant with the development of a CD4 $4^{+}$Th1 response cross-reactive with the native PLP139-151 epitope. In contrast, a virus expressing an amino acid substitution at the primary TCR contact site (W144A PLP139-BeAn) did not induce the early demyelinating disease consistent with the failure to induce a cross-reactive CD4 ${ }^{+} \mathrm{T}$ cell response to PLP139151. These findings from disease induced by the virus infection are consistent with earlier studies demonstrating that immunization of SJL mice with the W144A peptide in CFA was immunogenic. W144A primed a specific $\mathrm{CD}^{+} \mathrm{T}$ cell response but did not lead to clinical experimental autoimmune encephalomyelitis (EAE) as the $\mathrm{T}$ cells failed to cross-react with the native PLP139-151 epitope (23). In contrast, direct immunization with PLP139151 peptides carrying $\mathrm{H} \rightarrow \mathrm{L}$ or $\mathrm{H} \rightarrow A$ substitutions at position 147 induces EAE and $T$ cell responses cross-reactive with the native PLP139-151 peptide $(27,28)$.

Direct evidence that molecular mimicry can initiate virus-induced demyelination was provided by the finding that SJL mice infected with TMEV encoding a PLP139-151 epitope mimic from $H$. influenzae exhibited early-onset demyelinating disease and cross-reactive $\mathrm{CD}^{+} \mathrm{T}$ cell response to PLP139-151. This sequence, HI574-586, lies within the protease IV protein of $H$. influenzae and is involved in the degradation of proteins, peptides, and glycopeptides. It is particularly significant 
that HI574-586 shares only 6 of 13 amino acids, including the primary TCR (position 144) and MHC (position 145) contacts but not the secondary TCR contact (position 147), with PLP139-151. Most remarkably, HI574586 fails to induce EAE in SJL mice directly immunized multiple times with the peptide emulsified in CFA (27) (our unpublished results), but it was able to induce disease when immunization was followed with an additional suboptimal PLP139-151 immunization (27). That the H. influenzae epitope mimic was able to induce demyelinating disease when presented to the immune system in the context of the TMEV infection emphasizes the importance of the natural infection process in the initiation of molecular mimicry.

Numerous previous studies have addressed the potential of molecular mimic epitopes to initiate an autoimmune $\mathrm{T}$ cell response and to induce CNS autoimmune disease. The initial description of molecular mimicry showed that rabbits immunized with a peptide mimic derived from hepatitis $\mathrm{B}$ virus polymerase induced $\mathrm{T}$ cells cross-reactive with myelin basic protein (MBP) and lesions in the CNS (4). Further experiments showed that transgenic mice expressing nucleoprotein or glycoprotein of lymphocytic choriomeningitis virus (LCMV) developed mild CNS pathology upon intraperitoneal infection with LCMV (29). More recent studies have used peptides identified by scanning protein databases to identify proteins with similarities to myelin proteins. MBP peptide mimics from a number of bacterial and viral proteins were shown to activate human MBP-specific T cell clones isolated from MS patients (10). Similar studies were conducted with PLP epitope mimics from mouse viral and bacterial proteins that were shown to activate $\mathrm{T}$ cell hybridomas derived from SJL mice primed with PLP139-151 (27). Other approaches have shown that $T$ cells from SJL mice immunized with virusderived peptide mimic of the MBP87-99 epitope in CFA could transfer EAE to naive recipients following in vitro restimulation with the corresponding peptide, indicating that viral epitope-specific $T$ cells can survive in vivo and trigger autoimmune disease (30). In contrast, immunization with viral or bacterial peptide mimics in IFA protected mice from later induction of EAE by MBP peptide immunization, indicating that peptide mimics may also behave as TCR antagonists if administered under Th2 skewing conditions (31). In a further study, vaccination of mice with DNA encoding PLP or encephalitogenic epitopes of PLP, although not directly inducing EAE, could enhance the severity of disease upon later immunization with PLP139-151 in CFA (32).

The current study significantly advances the previous studies by using a natural infection model to elucidate the mechanisms of virus-induced molecular mimicry. As detailed above, previous studies have used immunization techniques to stimulate autoreactive $\mathrm{T}$ cells via molecular mimicry and then assessed the ability of these $T$ cells to induce EAE or affect ongoing clinical disease. The concept of molecular mimicry-induced autoimmunity requires a natural infection process wherein a cross- a

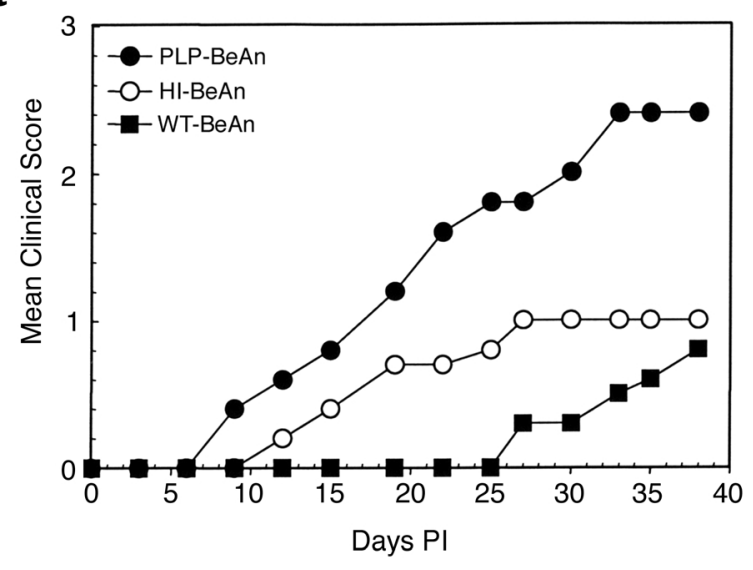

b

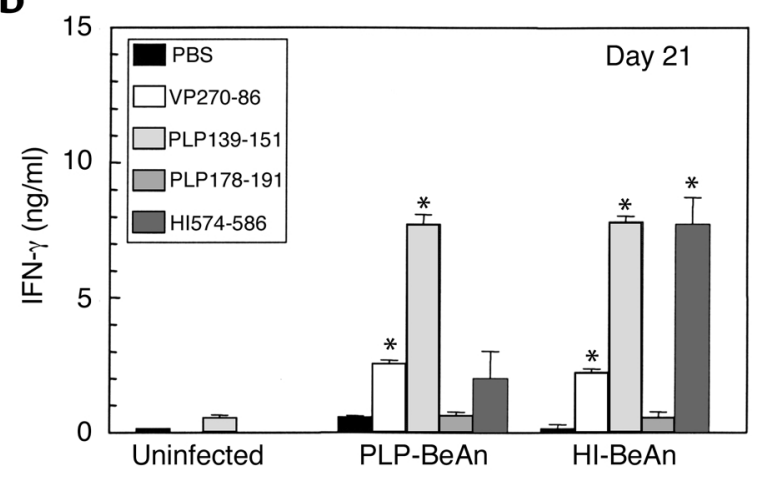

Figure 6

SJL mice infected with TMEV expressing a PLP139-151 molecular mimic from the protease IV protein of $H$. influenzae develop clinical disease associated with activation of PLP139-151-specific Th1 cells. Separate groups of mice $(n=8)$ were injected intracerebrally with $3 \times 10^{7}$ PFU of recombinant virus (PLP139-BeAn or HI-BeAn) or with wildtype BeAn (WT BeAn) and observed at varying times after infection for development of clinical signs (a). Clinical results are representative of two separate experiments. Spleen cells were harvested at 21 days $\mathrm{PI}$ and rechallenged with viral peptide (VP270-86), myelin peptides (PLP139-151, PLP178-191), or mimic peptide (HI574-586) and supernatants collected after 48 hours and measured for IFN- $\gamma$ secretion by ELISA (b). * Responses statistically significant compared with PBS-stimulated wells; $P \leq 0.01$.

reactive immune response capable of recognizing both the invading pathogen and the self epitope is induced. A major advantage of the current model is that the immune system encounters the mimic peptides in the context of infection with TMEV. Notable is the fact that TMEV expressing encephalitogenic PLP epitopes could induce early-onset disease in the absence of a stimulus associated with CFA, indicating that the virus itself is able to provide sufficient signals to the innate immune system to induce activation of the autoreactive T cells. In this regard, our recent evidence indicates that TMEV infection of quiescent microglia (macrophages and microglia are the primary targets of TMEV infection and persistence) results in upregulation of cytokines involved in the innate immune response (type I IFNs, TNF- $\alpha$, IL-6, IL-12, and IL-18). Virus-infected microglia also 
upregulated MHC class II and costimulatory molecules (B7-1, B7-2, CD40, and ICAM-1), which enabled the microglia to efficiently process and present both virus and myelin antigens to inflammatory $\mathrm{CD}^{+} \mathrm{Th} 1$ cells (J.K. Olson and S.D. Miller, manuscript submitted for publication). The primary host cell type infected by a virus encoding a potential self mimic epitope may be an important variable in determining whether an autoimmune disease is actually triggered. It has been reported that infection of SJL mice with a vaccinia virus construct expressing the entire coding region of PLP failed to induce CNS disease but rendered mice more susceptible to later induction of PLP139-151-induced EAE (33). Vaccinia infects many cell types, including B7 costimulatory molecule-deficient $B$ cells, which may have resulted in inefficient priming of PLP139-151-specific CD4 ${ }^{+}$ $\mathrm{T}$ cells. It is also significant that intravenous, subcutaneous, or intraperitoneal infection with the PLP139BeAn virus induces early-onset demyelinating disease, indicating that the initial site of infection can be in sites distal from the CNS (data not shown). This may indicate that the mimic-encoding pathogen does not need to infect the target organ of the autoimmune disease. However, these initial events during a viral infection play a significant part in the development of the molecular mimicry-induced autoimmune response, thus explaining why the same $H$. influenzae epitope mimic, when injected with CFA, did not result in disease (27). Another advantage of the current system is that the host is required to process the potential mimic epitope from a longer stretch of protein encoded by the virus, while previous studies have employed minimal peptide epitopes.

In summary, this study demonstrates a direct link between infection with a natural mouse pathogen and the development of CNS autoimmunity via molecular mimicry. Using appropriate transgenic mice (34), this virus model system should allow the functional identification of pathogen-encoded mimic epitopes for a variety of human myelin proteins potentially involved in the pathogenesis of MS.

\section{Acknowledgments}

This work is supported by NIH US Public Health Service grant NS-40460 and National Multiple Sclerosis Society grant RG3166-A-4. J.K. Olson is supported by NIH National Research Service Award grant NS-10893 and by Spinal Cord Research Foundation grant SCRF 2046.

1. Ota, K., et al. 1990. T-cell recognition of an immunodominant myelin basic protein epitope in multiple sclerosis. Nature. 346:183-187.

2. Bernard, C.C., and de Rosbo, N.K. 1991. Immunopathological recognition of autoantigens in multiple sclerosis. Acta Neurol. (Napoli). 13:171-178.

3. Kurtzke, J.F. 1993. Epidemiologic evidence for multiple sclerosis as an infection. Clin. Microbiol. Rev. 6:382-427.

4. Fujinami, R.S., and Oldstone, M.B. 1985. Amino acid homology between the encephalitogenic site of myelin basic protein and virus: mechanism for autoimmunity. Science. 230:1043-1045.

5. Oldstone, M.B. 1987. Molecular mimicry and autoimmune disease. Cell. 50:819-820.

6. Miller, S.D., and Karpus, W.J. 1994. The immunopathogenesis and regulation of T-cell mediated demyelinating diseases. Immunol. Today. 15:356-361.

7. McRae, B.L., Vanderlugt, C.L., Dal Canto, M.C., and Miller, S.D. 1995.
Functional evidence for epitope spreading in the relapsing pathology of experimental autoimmune encephalomyelitis. J. Exp. Med. 182:75-85.

8. Vanderlugt, C.L., Karandikar, N.J., Bluestone, J.A., and Miller, S.D. 1998

The functional significance of epitope spreading and its regulation by costimulatory interactions. Immunol. Rev. 164:63-72.

9. Scherer, M.T., Ignatowicz, L., Winslow, G.M., Kappler, J.W., and Marrack, P. 1993. Superantigens: bacterial and viral proteins that manipulate the immune system. Ann. Rev. Cell Biol. 9:101-128.

10. Wucherpfennig, K.W., and Strominger, J.L. 1995. Molecular mimicry in $\mathrm{T}$ cell-mediated autoimmunity: viral peptides activate human $\mathrm{T}$ cell clones specific for myelin basic protein. Cell. 80:695-705.

11. Ohashi, P.S., et al. 1991. Ablation of "tolerance" and induction of diabetes by virus infection in viral antigen transgenic mice. Cell. 65:305-317.

12. Oldstone, M.B., Nerenberg, M., Southern, P., Price, J., and Lewicki, H. 1991 Virus infection triggers insulin-dependent diabetes mellitus in a transgenic model: role of anti-self (virus) immune response. Cell. 65:319-331.

13. Hemmer, B., et al. 1997. Identification of high potency microbial and self ligands for a human autoreactive class II-restricted T cell clone. J. Exp. Med. 185:1651-1659.

14. Gran, B., Hemmer, B., Vergelli, M., McFarland, H.F., and Martin, R. 1999. Molecular mimicry and multiple sclerosis: degenerate T-cell recognition and the induction of autoimmunity. Ann. Neurol. 45:559-567.

15. Gross, D.M., et al. 1998. Identification of LFA-1 as a candidate autoantigen in treatment-resistant Lyme arthritis. Science. 281:703-706.

16. Hemmer, B., et al. 1999. Identification of candidate T-cell epitopes and molecular mimics in chronic Lyme disease. Nat. Med. 5:1375-1382.

17. Bachmaier, K., et al. 1999. Chlamydia infections and heart disease linked through antigenic mimicry. Science. 283:1335-1339.

18. Zhao, Z.-S., Granucci, F., Yeh, L., Schaffer, P.A., and Cantor, H. 1998. Molecular mimicry by herpes simplex virus-type 1: autoimmune disease after viral infection. Science. 279:1344-1347.

19. Lipton, H.L. 1975. Theiler's virus infection in mice: an unusual biphasic disease process leading to demyelination. Infect. Immun. 11:1147-1155.

20. Miller, S.D., and Gerety, S.J. 1990. Immunologic aspects of Theiler's murine encephalomyelitis virus (TMEV)-induced demyelinating disease. Seminars in Virology. 1:263-272.

21. Karpus, W.J., Pope, J.G., Peterson, J.D., Dal Canto, M.C., and Miller, S.D. 1995. Inhibition of Theiler's virus-mediated demyelination by peripheral immune tolerance induction. J. Immunol. 155:947-957.

22. Miller, S.D., et al. 1997. Persistent infection with Theiler's virus leads to CNS autoimmunity via epitope spreading. Nat. Med. 3:1133-1136.

23. McRae, B.L., and Miller, S.D. 1994. Fine specificity of $\mathrm{CD}^{+} \mathrm{T}$ cell responses to the dominant encephalitogenic PLP 139-151 peptide in SJL/J mice. Neurochem. Res. 19:997-1004.

24. Kuchroo, V.K., et al. 1994. A single TCR antagonist peptide inhibits experimental allergic encephalomyelitis mediated by a diverse $\mathrm{T}$ cell repertoire. J. Immunol. 153:3326-3336.

25. Katz-Levy, Y., et al. 2000. Temporal development of autoreactive Th1 responses and endogenous antigen presentation of self myelin epitopes by CNS-resident APCs in Theiler's virus-infected mice. J. Immunol. 165:5304-5314.

26. Pope, J.G., Karpus, W.J., Vanderlugt, C.L., and Miller, S.D. 1996. Flow cytometric and functional analyses of CNS-infiltrating cells in SJL/J mice with Theiler's virus-induced demyelinating disease: evidence for a $\mathrm{CD}^{+} \mathrm{T}$ cell-mediated pathology. J. Immunol. 156:4050-4058.

27. Carrizosa, A.M., et al. 1998. Expansion by self antigen is necessary for the induction of experimental autoimmune encephalomyelitis by $\mathrm{T}$ cells primed with a cross-reactive environmental antigen. J. Immunol. 161:3307-3314

28. McRae, B.L., Karandikar, N.J., and Miller, S.D. 1997. Degenerate antigen recognition by $\mathrm{CD}^{+}$effector $\mathrm{T}$ cells in experimental autoimmune encephalomyelitis. J. Neuroimmunol. 75:156-162.

29. Evans, C.F., Horwitz, M.S., Hobbs, M.V., and Oldstone, M.B. 1996. Viral infection of transgenic mice expressing a viral protein in oligodendrocytes leads to chronic central nervous system autoimmune disease. J. Exp. Med. 184:2371-2384.

30. Ufret-Vincenty, R.L., et al. 1998. In vivo survival of viral antigen-specific $\mathrm{T}$ cells that induce experimental autoimmune encephalomyelitis. J. Exp. Med. 188:1725-1738.

31. Ruiz, P.J., et al. 1999. Microbial epitopes act as altered peptide ligands to prevent experimental autoimmune encephalomyelitis. J. Exp. Med. 189:1275-1284.

32. Tsunoda, I., Kuang, L.Q., Tolley, N.D., Whitton, J.L., and Fujinami, R.S. 1998. Enhancement of experimental allergic encephalomyelitis (EAE) by DNA immunization with myelin proteolipid protein (PLP) plasmid DNA. J. Neuropathol. Exp. Neurol. 57:758-767.

33. Barnett, L.A., Whitton, J.L., Wada, Y., and Fujinami, R.S. 1993. Enhancement of autoimmune disease using recombinant vaccinia virus encoding myelin proteolipid protein. J. Neuroimmunol. 44:15-25.

34. Madsen, L.S., et al. 1999. A humanized model for multiple sclerosis using HLA-DR2 and a human T- cell receptor. Nat. Genet. 23:343-347. 